\title{
Power series method and approximate linear differential equations of second order
}

\author{
Soon-Mo Jung ${ }^{1}$ and Hamdullah Şevli ${ }^{2 *}$
}

"Correspondence:

hsevli@yahoo.com

${ }^{2}$ Department of Mathematics, Faculty of Sciences and Arts,

Istanbul Commerce University, Uskudar, Istanbul, 34672, Turkey Full list of author information is available at the end of the article

\begin{abstract}
In this paper, we will establish a theory for the power series method that can be applied to various types of linear differential equations of second order to prove the Hyers-Ulam stability.

MSC: Primary 34A05; 39B82; secondary 26D10; 34A40

Keywords: power series method; approximate linear differential equation; simple harmonic oscillator equation; Hyers-Ulam stability; approximation
\end{abstract}

\section{Introduction}

Let $X$ be a normed space over a scalar field $\mathbb{K}$, and let $I \subset \mathbb{R}$ be an open interval, where $\mathbb{K}$ denotes either $\mathbb{R}$ or $\mathbb{C}$. Assume that $a_{0}, a_{1}, \ldots, a_{n}: I \rightarrow \mathbb{K}$ and $g: I \rightarrow X$ are given continuous functions. If for every $n$ times continuously differentiable function $y: I \rightarrow X$ satisfying the inequality

$$
\left\|a_{n}(x) y^{(n)}(x)+a_{n-1}(x) y^{(n-1)}(x)+\cdots+a_{1}(x) y^{\prime}(x)+a_{0}(x) y(x)+g(x)\right\| \leq \varepsilon
$$

for all $x \in I$ and for a given $\varepsilon>0$, there exists an $n$ times continuously differentiable solution $y_{0}: I \rightarrow X$ of the differential equation

$$
a_{n}(x) y^{(n)}(x)+a_{n-1}(x) y^{(n-1)}(x)+\cdots+a_{1}(x) y^{\prime}(x)+a_{0}(x) y(x)+g(x)=0
$$

such that $\left\|y(x)-y_{0}(x)\right\| \leq K(\varepsilon)$ for any $x \in I$, where $K(\varepsilon)$ is an expression of $\varepsilon$ with $\lim _{\varepsilon \rightarrow 0} K(\varepsilon)=0$, then we say that the above differential equation has the Hyers-Ulam stability. For more detailed definitions of the Hyers-Ulam stability, we refer the reader to [1-8].

Obłoza seems to be the first author who has investigated the Hyers-Ulam stability of linear differential equations (see $[9,10])$. Thereafter, Alsina and Ger [11] proved the HyersUlam stability of the differential equation $y^{\prime}(x)=y(x)$. It was further proved by Takahasi $e t$ $a l$. that the Hyers-Ulam stability holds for the Banach space valued differential equation $y^{\prime}(x)=\lambda y(x)$ (see [12] and also [13-15]).

Moreover, Miura et al. [16] investigated the Hyers-Ulam stability of an $n$ th-order linear differential equation. The first author also proved the Hyers-Ulam stability of various linear differential equations of first order (ref. [17-25]).

Recently, the first author applied the power series method to studying the Hyers-Ulam stability of several types of linear differential equations of second order (see [26-34]). 
However, it was inconvenient that he had to alter and apply the power series method with respect to each differential equation in order to study the Hyers-Ulam stability. Thus, it is inevitable to develop a power series method that can be comprehensively applied to different types of differential equations.

In Sections 2 and 3 of this paper, we establish a theory for the power series method that can be applied to various types of linear differential equations of second order to prove the Hyers-Ulam stability.

Throughout this paper, we assume that the linear differential equation of second order of the form

$$
p(x) y^{\prime \prime}(x)+q(x) y^{\prime}(x)+r(x) y(x)=0,
$$

for which $x=0$ is an ordinary point, has the general solution $y_{h}:\left(-\rho_{0}, \rho_{0}\right) \rightarrow \mathbb{C}$, where $\rho_{0}$ is a constant with $0<\rho_{0} \leq \infty$ and the coefficients $p, q, r:\left(-\rho_{0}, \rho_{0}\right) \rightarrow \mathbb{C}$ are analytic at 0 and have power series expansions

$$
p(x)=\sum_{m=0}^{\infty} p_{m} x^{m}, \quad q(x)=\sum_{m=0}^{\infty} q_{m} x^{m} \quad \text { and } \quad r(x)=\sum_{m=0}^{\infty} r_{m} x^{m}
$$

for all $x \in\left(-\rho_{0}, \rho_{0}\right)$. Since $x=0$ is an ordinary point of $(1)$, we remark that $p_{0} \neq 0$.

\section{Inhomogeneous differential equation}

In the following theorem, we solve the linear inhomogeneous differential equation of second order of the form

$$
p(x) y^{\prime \prime}(x)+q(x) y^{\prime}(x)+r(x) y(x)=\sum_{m=0}^{\infty} a_{m} x^{m}
$$

under the assumption that $x=0$ is an ordinary point of the associated homogeneous linear differential equation (1).

Theorem 2.1 Assume that the radius of convergence of power series $\sum_{m=0}^{\infty} a_{m} x^{m}$ is $\rho_{1}>0$ and that there exists a sequence $\left\{c_{m}\right\}$ satisfying the recurrence relation

$$
\sum_{k=0}^{m}\left[(k+2)(k+1) c_{k+2} p_{m-k}+(k+1) c_{k+1} q_{m-k}+c_{k} r_{m-k}\right]=a_{m}
$$

for any $m \in \mathbb{N}_{0}$. Let $\rho_{2}$ be the radius of convergence of power series $\sum_{m=0}^{\infty} c_{m} x^{m}$ and let $\rho_{3}=\min \left\{\rho_{0}, \rho_{1}, \rho_{2}\right\}$, where $\left(-\rho_{0}, \rho_{0}\right)$ is the domain of the general solution to (1). Then every solution $y:\left(-\rho_{3}, \rho_{3}\right) \rightarrow \mathbb{C}$ of the linear inhomogeneous differential equation (2) can be expressed by

$$
y(x)=y_{h}(x)+\sum_{m=0}^{\infty} c_{m} x^{m}
$$

for all $x \in\left(-\rho_{3}, \rho_{3}\right)$, where $y_{h}(x)$ is a solution of the linear homogeneous differential equation (1). 
Proof Since $x=0$ is an ordinary point, we can substitute $\sum_{m=0}^{\infty} c_{m} x^{m}$ for $y(x)$ in (2) and use the formal multiplication of power series and consider (3) to get

$$
\begin{aligned}
p(x) & y^{\prime \prime}(x)+q(x) y^{\prime}(x)+r(x) y(x) \\
= & \sum_{m=0}^{\infty} \sum_{k=0}^{m} p_{m-k}(k+2)(k+1) c_{k+2} x^{m}+\sum_{m=0}^{\infty} \sum_{k=0}^{m} q_{m-k}(k+1) c_{k+1} x^{m} \\
& +\sum_{m=0}^{\infty} \sum_{k=0}^{m} r_{m-k} c_{k} x^{m} \\
= & \sum_{m=0}^{\infty} \sum_{k=0}^{m}\left[(k+2)(k+1) c_{k+2} p_{m-k}+(k+1) c_{k+1} q_{m-k}+c_{k} r_{m-k}\right] x^{m} \\
= & \sum_{m=0}^{\infty} a_{m} x^{m}
\end{aligned}
$$

for all $x \in\left(-\rho_{3}, \rho_{3}\right)$. That is, $\sum_{m=0}^{\infty} c_{m} x^{m}$ is a particular solution of the linear inhomogeneous differential equation (2), and hence every solution $y:\left(-\rho_{3}, \rho_{3}\right) \rightarrow \mathbb{C}$ of (2) can be expressed by

$$
y(x)=y_{h}(x)+\sum_{m=0}^{\infty} c_{m} x^{m}
$$

where $y_{h}(x)$ is a solution of the linear homogeneous differential equation (1).

For the most common case in applications, the coefficient functions $p(x), q(x)$, and $r(x)$ of the linear differential equation (1) are simple polynomials. In such a case, we have the following corollary.

Corollary 2.2 Let $p(x), q(x)$, and $r(x)$ be polynomials of degree at most $d \geq 0$. In particular, let $d_{0}$ be the degree of $p(x)$. Assume that the radius of convergence of power series $\sum_{m=0}^{\infty} a_{m} x^{m}$ is $\rho_{1}>0$ and that there exists a sequence $\left\{c_{m}\right\}$ satisfying the recurrence formula

$$
\sum_{k=m_{0}}^{m}\left[(k+2)(k+1) c_{k+2} p_{m-k}+(k+1) c_{k+1} q_{m-k}+c_{k} r_{m-k}\right]=a_{m}
$$

for any $m \in \mathbb{N}_{0}$, where $m_{0}=\max \{0, m-d\}$. If the sequence $\left\{c_{m}\right\}$ satisfies the following conditions:

(i) $\lim _{m \rightarrow \infty} c_{m-1} / m c_{m}=0$,

(ii) there exists a complex number $L$ such that $\lim _{m \rightarrow \infty} c_{m} / c_{m-1}=L$ and

$$
p_{d_{0}}+L p_{d_{0}-1}+\cdots+L^{d_{0}-1} p_{1}+L^{d_{0}} p_{0} \neq 0
$$

then every solution $y:\left(-\rho_{3}, \rho_{3}\right) \rightarrow \mathbb{C}$ of the linear inhomogeneous differential equation (2) can be expressed by

$$
y(x)=y_{h}(x)+\sum_{m=0}^{\infty} c_{m} x^{m}
$$


for all $x \in\left(-\rho_{3}, \rho_{3}\right)$, where $\rho_{3}=\min \left\{\rho_{0}, \rho_{1}\right\}$ and $y_{h}(x)$ is a solution of the linear homogeneous differential equation (1).

Proof Let $m$ be any sufficiently large integer. Since $p_{d+1}=p_{d+2}=\cdots=0, q_{d+1}=q_{d+2}=\cdots=$ 0 and $r_{d+1}=r_{d+2}=\cdots=0$, if we substitute $m-d+k$ for $k$ in (4), then we have

$$
\begin{aligned}
a_{m}= & \sum_{k=0}^{d}\left[(m-d+k+2)(m-d+k+1) c_{m-d+k+2} p_{d-k}\right. \\
& \left.+(m-d+k+1) c_{m-d+k+1} q_{d-k}+c_{m-d+k} r_{d-k}\right] .
\end{aligned}
$$

By (i) and (ii), we have

$$
\begin{aligned}
\limsup _{m \rightarrow \infty}\left|a_{m}\right|^{1 / m} & \limsup _{m \rightarrow \infty} \mid \sum_{k=0}^{d}(m-d+k+2)(m-d+k+1) c_{m-d+k+2} \\
& \times\left(p_{d-k}+\frac{q_{d-k}}{(m-d+k+2)} \frac{c_{m-d+k+1}}{c_{m-d+k+2}}\right. \\
& \left.+\frac{r_{d-k}}{(m-d+k+2)(m-d+k+1)} \frac{c_{m-d+k}}{c_{m-d+k+1}} \frac{c_{m-d+k+1}}{c_{m-d+k+2}}\right)\left.\right|^{1 / m} \\
= & \limsup _{m \rightarrow \infty}\left|\sum_{k=0}^{d}(m-d+k+2)(m-d+k+1) c_{m-d+k+2} p_{d-k}\right|^{1 / m} \\
= & \limsup _{m \rightarrow \infty}\left|\sum_{k=d-d_{0}}^{d}(m-d+k+2)(m-d+k+1) c_{m-d+k+2} p_{d-k}\right|^{1 / m} \\
= & \limsup _{m \rightarrow \infty}\left|\left(m-d_{0}+2\right)\left(m-d_{0}+1\right) c_{m-d_{0}+2}\left(p_{d_{0}}+L p_{d_{0}-1}+\cdots+L^{d_{0}} p_{0}\right)\right|^{1 / m} \\
= & \limsup _{m \rightarrow \infty}\left|\left(p_{d_{0}}+L p_{d_{0}-1}+\cdots+L^{d_{0}} p_{0}\right)\left(m-d_{0}+2\right)\left(m-d_{0}+1\right)\right|^{1 / m} \\
& \quad \times\left(\left|c_{m-d_{0}+2}\right|^{1 /\left(m-d_{0}+2\right)}\right)^{\left(m-d_{0}+2\right) / m} \\
= & \limsup _{m \rightarrow \infty}\left|c_{m-d_{0}+2}\right|^{1 /\left(m-d_{0}+2\right)},
\end{aligned}
$$

which implies that the radius of convergence of the power series $\sum_{m=0}^{\infty} c_{m} x^{m}$ is $\rho_{1}$. The rest of this corollary immediately follows from Theorem 2.1.

In many cases, it occurs that $p(x) \equiv 1$ in (1). For this case, we obtain the following corollary.

Corollary 2.3 Let $\rho_{3}$ be a distance between the origin 0 and the closest one among singular points of $q(z), r(z)$, or $\sum_{m=0}^{\infty} a_{m} z^{m}$ in a complex variable $z$. If there exists a sequence $\left\{c_{m}\right\}$ satisfying the recurrence relation

$$
(m+2)(m+1) c_{m+2}+\sum_{k=0}^{m}\left[(k+1) c_{k+1} q_{m-k}+c_{k} r_{m-k}\right]=a_{m}
$$


for any $m \in \mathbb{N}_{0}$, then every solution $y:\left(-\rho_{3}, \rho_{3}\right) \rightarrow \mathbb{C}$ of the linear inhomogeneous differential equation

$$
y^{\prime \prime}(x)+q(x) y^{\prime}(x)+r(x) y(x)=\sum_{m=0}^{\infty} a_{m} x^{m}
$$

can be expressed by

$$
y(x)=y_{h}(x)+\sum_{m=0}^{\infty} c_{m} x^{m}
$$

for all $x \in\left(-\rho_{3}, \rho_{3}\right)$, where $y_{h}(x)$ is a solution of the linear homogeneous differential equation (1) with $p(x) \equiv 1$.

Proof If we put $p_{0}=1$ and $p_{i}=0$ for each $i \in \mathbb{N}$, then the recurrence relation (3) reduces to (5). As we did in the proof of Theorem 2.1, we can show that $\sum_{m=0}^{\infty} c_{m} x^{m}$ is a particular solution of the linear inhomogeneous differential equation (6).

According to [35, Theorem 7.4] or [36, Theorem 5.2.1], there is a particular solution $y_{0}(x)$ of $(6)$ in a form of power series in $x$ whose radius of convergence is at least $\rho_{3}$. Moreover, since $\sum_{m=0}^{\infty} c_{m} x^{m}$ is a solution of (6), it can be expressed as a sum of both $y_{0}(x)$ and a solution of the homogeneous equation (1) with $p(x) \equiv 1$. Hence, the radius of convergence of $\sum_{m=0}^{\infty} c_{m} x^{m}$ is at least $\rho_{3}$.

Now, every solution $y:\left(-\rho_{3}, \rho_{3}\right) \rightarrow \mathbb{C}$ of (6) can be expressed by

$$
y(x)=y_{h}(x)+\sum_{m=0}^{\infty} c_{m} x^{m}
$$

where $y_{h}(x)$ is a solution of the linear differential equation (1) with $p(x) \equiv 1$.

\section{Approximate differential equation}

In this section, let $\rho_{1}>0$ be a constant. We denote by $\mathcal{C}$ the set of all functions $y$ : $\left(-\rho_{1}, \rho_{1}\right) \rightarrow \mathbb{C}$ with the following properties:

(a) $y(x)$ is expressible by a power series $\sum_{m=0}^{\infty} b_{m} x^{m}$ whose radius of convergence is at least $\rho_{1}$;

(b) There exists a constant $K \geq 0$ such that $\sum_{m=0}^{\infty}\left|a_{m} x^{m}\right| \leq K\left|\sum_{m=0}^{\infty} a_{m} x^{m}\right|$ for any $x \in\left(-\rho_{1}, \rho_{1}\right)$, where

$$
a_{m}=\sum_{k=0}^{m}\left[(k+2)(k+1) b_{k+2} p_{m-k}+(k+1) b_{k+1} q_{m-k}+b_{k} r_{m-k}\right]
$$

for all $m \in \mathbb{N}_{0}$ and $p_{0} \neq 0$.

Lemma 3.1 Given a sequence $\left\{a_{m}\right\}$, let $\left\{c_{m}\right\}$ be a sequence satisfying the recurrence formula (3) for all $m \in \mathbb{N}_{0}$. If $p_{0} \neq 0$ and $n \geq 2$, then $c_{n}$ is a linear combination of $a_{0}, a_{1}, \ldots, a_{n-2}, c_{0}$, and $c_{1}$. 
Proof We apply induction on $n$. Since $p_{0} \neq 0$, if we set $m=0$ in (3), then

$$
c_{2}=\frac{1}{2 p_{0}} a_{0}-\frac{r_{0}}{2 p_{0}} c_{0}-\frac{q_{0}}{2 p_{0}} c_{1}
$$

i.e., $c_{2}$ is a linear combination of $a_{0}, c_{0}$, and $c_{1}$. Assume now that $n$ is an integer not less than 2 and $c_{i}$ is a linear combination of $a_{0}, \ldots, a_{i-2}, c_{0}, c_{1}$ for all $i \in\{2,3, \ldots, n\}$, namely,

$$
c_{i}=\alpha_{i}^{0} a_{0}+\alpha_{i}^{1} a_{1}+\cdots+\alpha_{i}^{i-2} a_{i-2}+\beta_{i} c_{0}+\gamma_{i} c_{1},
$$

where $\alpha_{i}^{0}, \ldots, \alpha_{i}^{i-2}, \beta_{i}, \gamma_{i}$ are complex numbers. If we replace $m$ in (3) with $n-1$, then

$$
\begin{aligned}
a_{n-1}= & 2 c_{2} p_{n-1}+c_{1} q_{n-1}+c_{0} r_{n-1} \\
& +6 c_{3} p_{n-2}+2 c_{2} q_{n-2}+c_{1} r_{n-2} \\
& +\cdots \\
& +n(n-1) c_{n} p_{1}+(n-1) c_{n-1} q_{1}+c_{n-2} r_{1} \\
& +(n+1) n c_{n+1} p_{0}+n c_{n} q_{0}+c_{n-1} r_{0} \\
= & (n+1) n p_{0} c_{n+1}+\left[n(n-1) p_{1}+n q_{0}\right] c_{n}+\cdots \\
& +\left(2 p_{n-1}+2 q_{n-2}+r_{n-3}\right) c_{2}+\left(q_{n-1}+r_{n-2}\right) c_{1}+r_{n-1} c_{0},
\end{aligned}
$$

which implies

$$
\begin{aligned}
c_{n+1}= & \frac{1}{(n+1) n p_{0}} a_{n-1}-\frac{n(n-1) p_{1}+n q_{0}}{(n+1) n p_{0}} c_{n}-\cdots \\
& -\frac{2 p_{n-1}+2 q_{n-2}+r_{n-3}}{(n+1) n p_{0}} c_{2}-\frac{q_{n-1}+r_{n-2}}{(n+1) n p_{0}} c_{1}-\frac{r_{n-1}}{(n+1) n p_{0}} c_{0} \\
= & \alpha_{n+1}^{0} a_{0}+\alpha_{n+1}^{1} a_{1}+\cdots+\alpha_{n+1}^{n-1} a_{n-1}+\beta_{n+1} c_{0}+\gamma_{n+1} c_{1},
\end{aligned}
$$

where $\alpha_{n+1}^{0}, \ldots, \alpha_{n+1}^{n-1}, \beta_{n+1}, \gamma_{n+1}$ are complex numbers. That is, $c_{n+1}$ is a linear combination of $a_{0}, a_{1}, \ldots, a_{n-1}, c_{0}, c_{1}$, which ends the proof.

In the following theorem, we investigate a kind of Hyers-Ulam stability of the linear differential equation (1). In other words, we answer the question whether there exists an exact solution near every approximate solution of (1). Since $x=0$ is an ordinary point of (1), we remark that $p_{0} \neq 0$.

Theorem 3.2 Let $\left\{c_{m}\right\}$ be a sequence of complex numbers satisfying the recurrence relation (3) for all $m \in \mathbb{N}_{0}$, where (b) is referred for the value of $a_{m}$, and let $\rho_{2}$ be the radius of convergence of the power series $\sum_{m=0}^{\infty} c_{m} x^{m}$. Define $\rho_{3}=\min \left\{\rho_{0}, \rho_{1}, \rho_{2}\right\}$, where $\left(-\rho_{0}, \rho_{0}\right)$ is the domain of the general solution to (1). Assume that $y:\left(-\rho_{1}, \rho_{1}\right) \rightarrow \mathbb{C}$ is an arbitrary function belonging to $\mathcal{C}$ and satisfying the differential inequality

$$
\left|p(x) y^{\prime \prime}(x)+q(x) y^{\prime}(x)+r(x) y(x)\right| \leq \varepsilon
$$


for all $x \in\left(-\rho_{3}, \rho_{3}\right)$ and for some $\varepsilon>0$. Let $\alpha_{n}^{0}, \alpha_{n}^{1}, \ldots, \alpha_{n}^{n-2}, \beta_{n}, \gamma_{n}$ be the complex numbers satisfying

$$
c_{n}=\alpha_{n}^{0} a_{0}+\alpha_{n}^{1} a_{1}+\cdots+\alpha_{n}^{n-2} a_{n-2}+\beta_{n} c_{0}+\gamma_{n} c_{1}
$$

for any integer $n \geq 2$. If there exists a constant $C>0$ such that

$$
\left|\alpha_{n}^{0} a_{0}+\alpha_{n}^{1} a_{1}+\cdots+\alpha_{n}^{n-2} a_{n-2}\right| \leq C\left|a_{n}\right|
$$

for all integers $n \geq 2$, then there exists a solution $y_{h}:\left(-\rho_{3}, \rho_{3}\right) \rightarrow \mathbb{C}$ of the linear homogeneous differential equation (1) such that

$$
\left|y(x)-y_{h}(x)\right| \leq C K \varepsilon
$$

for all $x \in\left(-\rho_{3}, \rho_{3}\right)$, where $K$ is the constant determined in (b).

Proof By the same argument presented in the proof of Theorem 2.1 with $\sum_{m=0}^{\infty} b_{m} x^{m}$ instead of $\sum_{m=0}^{\infty} c_{m} x^{m}$, we have

$$
p(x) y^{\prime \prime}(x)+q(x) y^{\prime}(x)+r(x) y(x)=\sum_{m=0}^{\infty} a_{m} x^{m}
$$

for all $x \in\left(-\rho_{3}, \rho_{3}\right)$. In view of (b), there exists a constant $K \geq 0$ such that

$$
\sum_{m=0}^{\infty}\left|a_{m} x^{m}\right| \leq K\left|\sum_{m=0}^{\infty} a_{m} x^{m}\right|
$$

for all $x \in\left(-\rho_{1}, \rho_{1}\right)$.

Moreover, by using (7), (10), and (11), we get

$$
\sum_{m=0}^{\infty}\left|a_{m} x^{m}\right| \leq K\left|\sum_{m=0}^{\infty} a_{m} x^{m}\right| \leq K \varepsilon
$$

for any $x \in\left(-\rho_{3}, \rho_{3}\right)$. (That is, the radius of convergence of power series $\sum_{m=0}^{\infty} a_{m} x^{m}$ is at least $\rho_{3}$.)

According to Theorem 2.1 and (10), $y(x)$ can be written as

$$
y(x)=y_{h}(x)+\sum_{n=0}^{\infty} c_{n} x^{n}
$$

for all $x \in\left(-\rho_{3}, \rho_{3}\right)$, where $y_{h}(x)$ is a solution of the homogeneous differential equation (1). In view of Lemma 3.1, the $c_{n}$ can be expressed by a linear combination of the form (8) for each integer $n \geq 2$.

Since $\sum_{n=0}^{\infty} c_{n} x^{n}$ is a particular solution of (2), if we set $c_{0}=c_{1}=0$, then it follows from (8), (9), and (12) that

$$
\left|y(x)-y_{h}(x)\right| \leq \sum_{n=0}^{\infty}\left|c_{n} x^{n}\right| \leq C K \varepsilon
$$

for all $x \in\left(-\rho_{3}, \rho_{3}\right)$. 


\section{Competing interests}

The authors declare that they have no competing interests.

\section{Authors' contributions}

The authors declare that this paper is their original paper. All authors read and approved the final manuscript.

\section{Author details}

${ }^{1}$ Mathematics Section, College of Science and Technology, Hongik University, Sejong, 339-701, Republic of Korea. ${ }^{2}$ Department of Mathematics, Faculty of Sciences and Arts, Istanbul Commerce University, Uskudar, Istanbul, 34672, Turkey.

\section{Acknowledgements}

Dedicated to Professor Hari M Srivastava.

This research was completed with the support of The Scientific and Technological Research Council of Turkey while the first author was a visiting scholar at Istanbul Commerce University, Istanbul, Turkey.

\section{Received: 14 December 2012 Accepted: 4 March 2013 Published: 26 March 2013}

\section{References}

1. Brillouet-Belluot, N, Brzdęk, J, Cieplinski, K: On some recent developments in Ulam's type stability. Abstr. Appl. Anal. 2012, Article ID 716936 (2012)

2. Czerwik, S: Functional Equations and Inequalities in Several Variables. World Scientific, River Edge (2002)

3. Hyers, DH: On the stability of the linear functional equation. Proc. Natl. Acad. Sci. USA 27, $222-224$ (1941)

4. Hyers, DH, Isac, G, Rassias, TM: Stability of Functional Equations in Several Variables. Birkhäuser, Boston (1998)

5. Hyers, DH, Rassias, TM: Approximate homomorphisms. Aequ. Math. 44, 125-153 (1992)

6. Jung, S-M: Hyers-Ulam-Rassias Stability of Functional Equations in Nonlinear Analysis. Springer, New York (2011)

7. Rassias, TM: On the stability of the linear mapping in Banach spaces. Proc. Am. Math. Soc. 72, 297-300 (1978)

8. Ulam, SM: Problems in Modern Mathematics. Wiley, New York (1964)

9. Obłoza, M: Hyers stability of the linear differential equation. Rocznik Nauk.-Dydakt. Prace Mat. 13, $259-270$ (1993)

10. Obłoza, M: Connections between Hyers and Lyapunov stability of the ordinary differential equations. Rocznik Nauk.-Dydakt. Prace Mat. 14, 141-146 (1997)

11. Alsina, C, Ger, R: On some inequalities and stability results related to the exponential function. J. Inequal. Appl. 2, 373-380 (1998)

12. Takahasi, S-E, Miura, T, Miyajima, S: On the Hyers-Ulam stability of the Banach space-valued differential equation $y^{\prime}=\lambda y$. Bull. Korean Math. Soc. 39, 309-315 (2002)

13. Miura, T: On the Hyers-Ulam stability of a differentiable map. Sci. Math. Jpn. 55, 17-24 (2002)

14. Miura, T, Jung, S-M, Takahasi, S-E: Hyers-Ulam-Rassias stability of the Banach space valued differential equations $y^{\prime}=\lambda y$. J. Korean Math. Soc. 41, 995-1005 (2004)

15. Miura, T, Miyajima, S, Takahasi, S-E: A characterization of Hyers-Ulam stability of first order linear differential operators. J. Math. Anal. Appl. 286, 136-146 (2003)

16. Miura, T, Miyajima, S, Takahasi, S-E: Hyers-Ulam stability of linear differential operator with constant coefficients. Math Nachr. 258, 90-96 (2003)

17. Cimpean, DS, Popa, D: On the stability of the linear differential equation of higher order with constant coefficients. Appl. Math. Comput. 217(8), 4141-4146 (2010)

18. Cimpean, DS, Popa, D: Hyers-Ulam stability of Euler's equation. Appl. Math. Lett. 24(9), 1539-1543 (2011)

19. Jung, S-M: Hyers-Ulam stability of linear differential equations of first order. Appl. Math. Lett. 17, 1135-1140 (2004)

20. Jung, S-M: Hyers-Ulam stability of linear differential equations of first order, II. Appl. Math. Lett. 19, 854-858 (2006)

21. Jung, S-M: Hyers-Ulam stability of linear differential equations of first order, III. J. Math. Anal. Appl. 311, 139-146 (2005)

22. Jung, S-M: Hyers-Ulam stability of a system of first order linear differential equations with constant coefficients. J. Math. Anal. Appl. 320, 549-561 (2006)

23. Lungu, N, Popa, D: On the Hyers-Ulam stability of a first order partial differential equation. Carpath. J. Math. 28(1), 77-82 (2012)

24. Lungu, N, Popa, D: Hyers-Ulam stability of a first order partial differential equation. J. Math. Anal. Appl. 385(1), 86-91 (2012)

25. Popa, D, Rasa, I: The Frechet functional equation with application to the stability of certain operators. J. Approx Theory 164(1), 138-144 (2012)

26. Jung, S-M: Legendre's differential equation and its Hyers-Ulam stability. Abstr. Appl. Anal. 2007, Article ID 56419 (2007). doi:10.1155/2007/56419

27. Jung, S-M: Approximation of analytic functions by Airy functions. Integral Transforms Spec. Funct. 19(12), 885-891 (2008)

28. Jung, S-M: Approximation of analytic functions by Hermite functions. Bull. Sci. Math. 133, 756-764 (2009)

29. Jung, S-M: Approximation of analytic functions by Legendre functions. Nonlinear Anal. 71(12), e103-e108 (2009)

30. Jung, S-M: Hyers-Ulam stability of differential equation $y^{\prime \prime}+2 x y^{\prime}-2 n y=0$. J. Inequal. Appl. 2010, Article ID 793197 (2010). doi:10.1155/2010/793197

31. Jung, S-M: Approximation of analytic functions by Kummer functions. J. Inequal. Appl. 2010, Article ID 898274 (2010). doi:10.1155/2010/898274

32. Jung, S-M: Approximation of analytic functions by Laguerre functions. Appl. Math. Comput. 218(3), 832-835 (2011). doi:10.1016/j.amc.2011.01.086

33. Jung, S-M, Rassias, TM: Approximation of analytic functions by Chebyshev functions. Abstr. Appl. Anal. 2011, Article ID 432961 (2011). doi:10.1155/2011/432961

34. Kim, B, Jung, S-M: Bessel's differential equation and its Hyers-Ulam stability. J. Inequal. Appl. 2007, Article ID 21640 (2007). doi:10.1155/2007/21640

35. Ross, CC: Differential Equations - An Introduction with Mathematica. Springer, New York (1995)

36. Kreyszig, E: Advanced Engineering Mathematics, 9th edn. Wiley, New York (2006) 
doi:10.1186/1687-1847-2013-76

Cite this article as: Jung and Şevli: Power series method and approximate linear differential equations of second order. Advances in Difference Equations 2013 2013:76.

Submit your manuscript to a SpringerOpen ${ }^{\circ}$ journal and benefit from:

- Convenient online submission

- Rigorous peer review

- Immediate publication on acceptance

- Open access: articles freely available online

- High visibility within the field

- Retaining the copyright to your article

Submit your next manuscript at $\gg$ springeropen.com 